\title{
EXPLICIT EVALUATION OF EULER SUMS
}

\section{by DAVID BORWEIN, JONATHAN M. BORWEIN and ROLAND GIRGENSOHN}

(Received 30th August 1993)

In response to a letter from Goldbach, Euler considered sums of the form

$$
\sigma_{b}(s, t):=\sum_{n=1}^{\infty}\left(1+\frac{1}{2^{s}}+\cdots+\frac{1}{(n-1)^{s}}\right) n^{-t},
$$

where $s$ and $t$ are positive integers.

As Euler discovered by a process of extrapolation (from $s+t \leqq 13), \sigma_{\mathrm{h}}(s, t)$ can be evaluated in terms of Riemann $\zeta$-functions when $s+t$ is odd. We provide a rigorous proof of Euler's discovery and then give analogous evaluations with proofs for corresponding alternating sums. Relatedly we give a formula for the series

$$
\sum_{n=1}^{\infty}\left(1+\frac{1}{2}+\cdots+\frac{1}{n}\right)^{2}(n+1)^{-m}
$$

This evaluation involves $\zeta$-functions and $\sigma_{\mathrm{h}}(2, m)$.

1991 Mathematics subject clasification: Primary 40A25, 40B05. Secondary 11M99, 33E99.

\section{Introduction}

We will use the usual definitions and identities involving the Riemann zeta function $\zeta$, the beta function $\beta$, the gamma function $\Gamma$, and the digamma or psi function $\Psi$; especially

$$
\beta(x, y):=\int_{0}^{1}(1-u)^{x-1} u^{y-1} d u=\frac{\Gamma(x) \Gamma(y)}{\Gamma(x+y)} \text { for } x>0, y>0
$$

(see [11, Theorem 7.69)] and

$$
\Psi(z):=\frac{d}{d z} \ln \Gamma(z)=-\gamma-\sum_{n=1}^{\infty}(-1)^{n} \zeta(n+1)(z-1)^{n} \text { for }|z-1|<1
$$

(see [11, Theorem 7.71]). 
Define

$$
s_{\mathrm{b}}(n, m):=\sum_{k=1}^{\infty} \frac{\left(1+\frac{1}{2}+\cdots+\frac{1}{k}\right)^{n}}{(k+1)^{m}} \text { for } n=1,2, \ldots ; m=2,3, \ldots
$$

and

$$
\sigma_{\mathrm{h}}(s, t):=\sum_{n=1}^{\infty} \frac{1}{n^{t}} \sum_{k=1}^{n-1} \frac{1}{k^{s}} \text { for } s=1,2, \ldots ; t=2,3, \ldots
$$

In this paper, we will consider $s_{\mathrm{b}}(2, m)$, leaving discussion of the more general $s_{\mathrm{b}}(n, m)$ for [1]. The purpose of this paper is to give a complete proof of the formulas

$$
\begin{aligned}
s_{\mathrm{h}}(2, m)= & \frac{m(m+1)}{3} \zeta(m+2)+\zeta(2) \zeta(m)-\frac{m^{m}}{2} \sum_{k=0}^{m-2} \zeta(m-k) \zeta(k+2) \\
& +\frac{1}{3} \sum_{k=2}^{m-2} \zeta(m-k) \sum_{j=1}^{k-1} \zeta(j+1) \zeta(k+1-j)+\sigma_{\mathrm{h}}(2, m)
\end{aligned}
$$

and, for $s+t=2 n+1, n \geqq 1$,

$$
\begin{aligned}
\sigma_{\mathrm{h}}(s, t)= & \frac{1}{2}\left[\left(\begin{array}{c}
s+t \\
s
\end{array}\right)-1\right] \zeta(s+t)+\zeta(s) \zeta(t) \\
& -\sum_{j=1}^{n}\left[\left(\begin{array}{c}
2 j-2 \\
s-1
\end{array}\right)+\left(\begin{array}{c}
2 j-2 \\
t-1
\end{array}\right)\right] \zeta(2 j-1) \zeta(s+t-2 j+1) \quad \text { if } s \text { is odd, } \\
\sigma_{\mathrm{h}}(s, t)= & -\frac{1}{2}\left[\left(\begin{array}{c}
s+t \\
s
\end{array}\right)+1\right] \zeta(s+t) \\
& +\sum_{j=1}^{n}\left[\left(\begin{array}{c}
2 j-2 \\
s-1
\end{array}\right)+\left(\begin{array}{c}
2 j-2 \\
t-1
\end{array}\right)\right] \zeta(2 j-1) \zeta(s+t-2 j+1) \quad \text { if } s \text { is even. }
\end{aligned}
$$

This last evaluation is due to Euler (cf. [5]); the terms involving $\zeta(1)$ which he used here can be cancelled formally if $t>1$. (Note that in the last two occurrences of this formula in Euler's paper almost all the signs are wrong.) Euler obtained his evaluation by computing many examples $(s+t \leqq 13)$ and then extrapolating the general formula, without actual proof. He proved, however, that

$$
2 \sigma_{\mathrm{h}}(1, m)=m \zeta(m+1)-\sum_{k=1}^{m-2} \zeta(m-k) \zeta(k+1) \quad \text { for all } m=2,3, \ldots
$$


In N. Nielsen's book [8, pp. 47-49], a clean version of Euler's proof for (3) can be found. In the present paper, we will give a complete proof of Euler's evaluation of $\sigma_{\mathrm{b}}(s, t)$, using a method which is closely related to Euler's.

It follows that

$$
\begin{aligned}
\sigma_{\mathrm{h}}(2,2 n-1)= & -\frac{2 n^{2}+n+1}{2} \zeta(2 n+1)+\zeta(2) \zeta(2 n-1) \\
& +\sum_{k=1}^{n-1} 2 k \zeta(2 k+1) \zeta(2 n-2 k) .
\end{aligned}
$$

From this and formula (2) we get

$$
\begin{aligned}
s_{\mathrm{h}}(2,2 n-1)= & \frac{2 n^{2}-7 n-3}{6} \zeta(2 n+1)+\zeta(2) \zeta(2 n-1)-\frac{1}{2} \sum_{k=1}^{n-2}(2 k-1) \zeta(2 n-1-2 k) \zeta(2 k+2) \\
& +\frac{1}{3} \sum_{k=1}^{n-2} \zeta(2 k+1) \sum_{j=1}^{n-2-k} \zeta(2 j+1) \zeta(2 n-1-2 k-2 j) .
\end{aligned}
$$

To see this, we need the identity

$$
\sum_{j=1}^{n-1} \zeta(2 j) \zeta(2 n-2 j)=\frac{2 n+1}{2} \zeta(2 n)
$$

which is well-known (see, e.g., formula (4), p. 49, in [8]) and can be proved with the use of the generating function of $\zeta(2 n) / \pi^{2 n}$, namely

$$
h(t):=\sum_{n=1}^{\infty} \zeta(2 n)(t / \pi)^{2 n}=\frac{1}{2}-\frac{t}{2} \cot t
$$

on observing that $h$ satisfies

$$
h^{2}-\frac{1}{2}(t h)^{\prime}=-\frac{1}{4} t^{2}
$$

Note that the last sum in this evaluation may be written as

$$
\sum_{\substack{a+b+c=2 n+1 \\ a, b, c>1, \text { odd }}} \zeta(a) \zeta(b) \zeta(c) .
$$

We also have 


$$
\begin{aligned}
& \sigma_{h}(2,2)=\frac{1}{2} \zeta^{2}(2)-\frac{1}{2} \zeta(4)=\frac{\pi^{4}}{120} \\
& \sigma_{h}(2,4)=-6 \zeta(6)+\frac{8}{3} \zeta(2) \zeta(4)+\zeta^{2}(3)=\zeta^{2}(3)-\frac{4 \pi^{6}}{2835},
\end{aligned}
$$

whereas the values $\sigma_{h}(2,2 n)$ for higher values of $n$ are not as easy to obtain. In fact, we have reason to believe (with very high probability) that these series are not expressible in terms of zeta functions alone; cf. [1]. From the previous two evaluations together with (1), however, we get that

$$
\begin{aligned}
& s_{\mathrm{h}}(2,2)=\frac{3}{2} \zeta(4)+\frac{1}{2} \zeta^{2}(2)=\frac{11}{4} \zeta(4), \\
& s_{\mathrm{h}}(2,4)=\frac{2}{3} \zeta(6)-\frac{1}{3} \zeta(2) \zeta(4)+\frac{1}{3} \zeta^{3}(2)-\zeta^{2}(3) .
\end{aligned}
$$

Series of the type $\sigma_{\mathrm{h}}$ and their alternating analogues have been studied previously; often the authors were not aware of what Euler had done. For further references see [2, p. 252]. In [4] and in [3], $s_{\mathrm{h}}(2,2)$ is evaluated by various methods (in other words, identity (2) is proved for $m=2$ ).

In the last section of this paper, we will give analogous formulas for the alternating series

$$
\begin{gathered}
\alpha_{\mathrm{h}}(s, t):=\sum_{n=1}^{\infty} \frac{(-1)}{n^{t}} \sum_{k=1}^{n-1} \frac{1}{k^{s}} \text { for } s=1,2, \ldots ; t=1,2, \ldots, \\
\sigma_{\mathrm{a}}(s, t):=\sum_{n=1}^{\infty} \frac{1}{n^{t}} \sum_{k=1}^{n-1} \frac{(-1)^{k-1}}{k^{s}} \text { for } s=1,2, \ldots ; t=2,3, \ldots, \\
\alpha_{\mathrm{a}}(s, t):=\sum_{n=1}^{\infty} \frac{(-1)^{n}}{n^{t}} \sum_{k=1}^{n-1} \frac{(-1)^{k-1}}{k^{s}} \text { for } s=1,2, \ldots ; t=1,2, \ldots
\end{gathered}
$$

\section{Proof of identity (1)}

We make use of the following identity. Since

$$
\int_{0}^{1} t^{n} \ln ^{m-1}(t) d t=(-1)^{m-1} \frac{(m-1) !}{(n+1)^{m}} \text { for } n>-1, m=1,2, \ldots
$$

we have for $f(t):=\sum_{n=0}^{\infty} a_{n} t^{n}(|t|<1)$ that 


$$
\frac{(-1)^{m-1}}{(m-1) !} \int_{0}^{1} \ln ^{m-1}(t) f(t) d t=\sum_{n=0}^{\infty} \frac{a_{n}}{(n+1)^{m}}
$$

provided that the final series is absolutely convergent.

With the use of (4), identity (2) and Euler's identity (3) can be proved in a unified way. We will therefore prove both of them here, but remind the reader that Euler and Nielsen gave an algebraic (integral-free) proof of (3). We need the following easily verified generating functions:

$$
\sum_{n=1}^{\infty}\left(1+\frac{1}{2}+\cdots+\frac{1}{n}\right) t^{n}=-\frac{\ln (1-t)}{1-t}
$$

and

$$
\sum_{n=1}^{\infty}\left(1+\frac{1}{2}+\cdots+\frac{1}{n}\right)^{2} t^{n}=\frac{\ln ^{2}(1-t)+\mathrm{Li}_{2}(t)}{1-t}
$$

where the dilogarithm $\mathrm{Li}_{2}(t):=\sum_{n=1}^{\infty} t^{n} / n^{2}$. A good reference for polylogarithms is [7].

We therefore have, on using (4) and integrating by parts,

$$
\begin{aligned}
\sigma_{\mathrm{h}}(1, m) & =\frac{(-1)^{m}}{(m-1) !} \int_{0}^{1} \frac{\ln ^{m-1}(t) \ln (1-t)}{1-t} d t \\
& =\frac{(-1)^{m}}{2(m-1) !} \int_{0}^{1} \frac{(m-1) \ln ^{m-2}(t) \ln ^{2}(1-t)}{t} d t \\
& =\frac{(-1)^{m}}{2(m-2) !} B_{1}^{(m-2)}(0)
\end{aligned}
$$

where

$$
\begin{gathered}
B_{1}(y):=\left.\frac{\partial^{2}}{\partial x^{2}} \beta(x, y)\right|_{x=1} . \text { Since } \\
\frac{\partial^{2}}{\partial x^{2}} \beta(x, y)=\beta(x, y)\left((\Psi(x)-\Psi(x+y))^{2}+\left(\Psi^{\prime}(x)-\Psi^{\prime}(x+y)\right)\right)
\end{gathered}
$$

we have

$$
B_{1}(y)=\frac{1}{y}\left((-\gamma-\Psi(y+1))^{2}+\left(\zeta(2)-\Psi^{\prime}(y+1)\right)\right)
$$


From the following two identities

$$
\begin{aligned}
(-\gamma-\Psi(y+1))^{2} & =\left(\sum_{m=1}^{\infty}(-1)^{m} \zeta(m+1) y^{m}\right)^{2} \\
& =\sum_{m=1}^{\infty}(-1)^{m} \sum_{k=1}^{m-1} \zeta(k+1) \zeta(m-k+1) y^{m}, \\
\zeta(2)-\Psi^{\prime}(y+1) & =\sum_{m=1}^{\infty}(-1)^{m+1}(m+1) \zeta(m+2) y^{m},
\end{aligned}
$$

which follow from (1), we get

$$
\begin{aligned}
\sum_{m=2}^{\infty}(-1)^{m} 2 \sigma_{\mathrm{h}}(1, m) y^{m-2}=\sum_{m=2}^{\infty} \frac{1}{(m-2) !} B_{1}^{(m-2)}(0) y^{m-2}=B_{1}(y) \\
\quad=\sum_{m=1}^{\infty}(-1)^{m-1}\left((m+1) \zeta(m+2)-\sum_{k=1}^{m-1} \zeta(k+1) \zeta(m-k+1)\right) y^{m-1}
\end{aligned}
$$

by (5). From this we get identity (3).

Similarly, we have, again on using (4) and integrating by parts,

$$
\begin{aligned}
s_{\mathrm{h}}(2, m) & =\frac{(-1)^{m-1}}{(m-1) !} \int_{0}^{1} \frac{\ln ^{m-1}(t)\left[\ln ^{2}(1-t)+\mathrm{Li}_{2}(t)\right]}{1-t} d t \\
& =\frac{(-1)^{m-1}}{3(m-1) !} \int_{0}^{1} \frac{(m-1) \ln ^{m-2}(t) \ln ^{3}(1-t)}{t} d t+\sum_{n=1}^{\infty} \frac{1+\frac{1}{2^{2}}+\cdots+\frac{1}{n^{2}}}{(n+1)^{m}} \\
& =\frac{(-1)^{m-1}}{3(m-2) !} B_{2}^{(m-2)}(0)+\sigma_{\mathrm{h}}(2, m),
\end{aligned}
$$

where

$$
B_{2}(y):=\left.\frac{\partial^{3}}{\partial x^{3}} \beta(x, y)\right|_{x=1}
$$

Since

$$
\begin{aligned}
\frac{\partial^{3}}{\partial x^{3}} \beta(x, y) & =\beta(x, y)\left((\Psi(x)-\Psi(x+y))^{3}+3(\Psi(x)-\Psi(x+y)) \cdot\left(\Psi^{\prime}(x)-\Psi^{\prime}(x+y)\right)\right. \\
& \left.+\left(\Psi^{\prime \prime}(x)-\Psi^{\prime \prime}(x+y)\right)\right),
\end{aligned}
$$


we have

$$
\begin{aligned}
B_{2}(y)= & (-\gamma-\Psi(y+1)) \cdot B_{1}(y) \\
& +2 \frac{1}{y}(-\gamma-\Psi(y+1)) \cdot\left(\zeta(2)-\Psi^{\prime}(y+1)\right)+\left(-2 \zeta(3)-\Psi^{\prime \prime}(y+1)\right) .
\end{aligned}
$$

From the identity

$$
-2 \zeta(3)-\Psi^{\prime \prime}(y+1)=\sum_{m=1}^{\infty}(-1)^{m}(m+1)(m+2) \zeta(m+3) y^{m}
$$

which again follows from (1), we get

$$
\begin{array}{rl}
\sum_{m=2}^{\infty}(-1)^{m-1} & 3\left(s_{\mathrm{h}}(2, m)-\sigma_{\mathrm{h}}(2, m)\right) y^{m-2} \\
= & \sum_{m=2}^{\infty} \frac{1}{(m-2) !} B_{2}^{(m-2)}(0) y^{m-2}=B_{2}(y) \\
= & \sum_{m=1}^{\infty}(-1)^{m} 2 \sum_{k=1}^{m} \zeta(k+1) \sigma_{\mathrm{h}}(1, m-k+2) y^{m} \\
& +\sum_{m=1}^{\infty}(-1)^{m+1} 2 \sum_{k=1}^{m-1}(m-k+1) \zeta(k+1) \zeta(m-k+2) y^{m-1} \\
& +\sum_{m=1}^{\infty}(-1)^{m}(m+1)(m+2) \zeta(m+3) y^{m-1}
\end{array}
$$

by (6). From this we get identity (2); note that

$$
\sum_{k=1}^{m-2}(m-k) \zeta(k+1) \zeta(m-k+1)=\frac{m}{2} \sum_{k=1}^{m-1} \zeta(k+1) \zeta(m-k+1)-\zeta(m) \zeta(2) .
$$

\section{Proof of Euler's formula}

Euler computed his examples of $\sigma_{\mathrm{h}}(s, t)$ by finding and solving linear equations between these quantities if $s+t=N$, a constant. We will follow the same method here, although we will use slightly different equations.

We need the following lemma which was known already to Euler (it can be proved by induction on $s+t$ ).

\section{Lemma 1. Define}




$$
A_{j}^{(s, t)}:=\left(\begin{array}{c}
s+t-j-1 \\
s-j
\end{array}\right) \text { and } B_{j}^{(s, t)}:=\left(\begin{array}{c}
s+t-j-1 \\
t-j
\end{array}\right)
$$

Then we have the partial fraction decomposition

$$
\frac{1}{x^{s}(1-x)^{t}}=\sum_{j=1}^{s} \frac{A_{j}^{(s, t)}}{x^{j}}+\sum_{j=1}^{t} \frac{B_{j}^{(s, t)}}{(1-x)^{j}}
$$

for $s, t \geqq 0, s+t \geqq 1$.

We will now derive systems of linear equations for the values $\sigma_{\mathrm{b}}(s, t)$ where $s+t=N$, a constant.

First, there is a simple relation between $\sigma_{\mathrm{h}}(s, t)$ and $\sigma_{\mathrm{h}}(t, s)$. This is due to Euler.

$$
\begin{aligned}
\sigma_{\mathrm{h}}(s, t) & =\sum_{n=1}^{\infty} \sum_{k=1}^{n-1} \frac{1}{k^{s}} \frac{1}{n^{2}}=\sum_{k=1}^{\infty} \sum_{n=k+1}^{\infty} \frac{1}{n^{t}} \frac{1}{k^{s}} \\
& =\sum_{k=1}^{\infty} \sum_{n=1}^{\infty} \frac{1}{n^{t} k^{s}}-\sum_{k=1}^{\infty} \sum_{n=1}^{k-1} \frac{1}{n^{t} k^{s}}-\sum_{k=1}^{\infty} \frac{1}{k^{s+t}} \\
& =\zeta(s) \zeta(t)-\sigma_{\mathrm{b}}(t, s)-\zeta(s+t),
\end{aligned}
$$

or

$$
\sigma_{\mathrm{h}}(s, t)+\sigma_{\mathrm{h}}(t, s)=\zeta(s) \zeta(t)-\zeta(s+t)
$$

for $s, t \geqq 2$. We will refer to these equations as "reflection formulas". It follows that $2 \sigma_{h}(s, s)=\zeta^{2}(s)-\zeta(2 s)$. In [9], corresponding reflection formulas have been found for triple series; recently, $M$. Hoffman gave a quite surprising generalization to multiple series ([6]).

Second, we have

$$
\begin{aligned}
\zeta(s) \zeta(t) & =\left(\sum_{k=1}^{\infty} \frac{1}{k^{s}}\right) \cdot\left(\sum_{n=1}^{\infty} \frac{1}{n^{t}}\right)=\sum_{n=1}^{\infty} \sum_{k=1}^{n-1} \frac{1}{k^{s}(n-k)^{t}} \\
& =\sum_{n=1}^{\infty} \sum_{k=1}^{n-1}\left(\sum_{j=1}^{s} \frac{A_{j}^{(s, t)}}{n^{s+t-j} k^{j}}+\sum_{j=1}^{t} \frac{B_{j}^{(s, t)}}{n^{s+t-j}(n-k)^{j}}\right) \\
& =\sum_{j=1}^{s} A_{j}^{(s, t)} \sigma_{\mathrm{h}}(j, s+t-j)+\sum_{j=1}^{t} B_{j}^{(s, t)} \sigma_{\mathrm{h}}(j, s+t-j),
\end{aligned}
$$


for $s, t \geqq 2$, with $A_{j}^{(s, t)}$ and $B_{j}^{(s, t)}$ defined as in Lemma 1 . We will refer to these equations as "decomposition formulas."

Euler used slightly different equations in place of the decomposition formulas, namely

$$
\sigma_{\mathrm{h}}(s, t)=(-1)^{s} \sum_{j=1}^{s}(-1)^{j} A_{j}^{(s, t)} \zeta(j) \zeta(s+t-j)+(-1)^{s} \sum_{j=1}^{t} B_{j}^{(s, t)} \sigma_{\mathrm{h}}(s+t-j, j) .
$$

These involve the infinite terms $\zeta(1)$ and $\sigma_{\mathrm{b}}(s+t-1,1)$. However, in his book [8], pp. 48-49], Nielsen used the following version of Euler's equations, together with the reflection formulas, to prove Euler's formula (3) algebraically:

$$
\begin{aligned}
\sigma_{\mathrm{h}}(s, t)= & (-1)^{s} \sum_{j=2}^{s}(-1)^{j} A_{j}^{(s, t)} \zeta(j) \zeta(s+t-j)+(-1)^{s} \sum_{j=2}^{t} B_{j}^{(s, t)} \sigma_{\mathrm{h}}(s+t-j, j) \\
& +(-1)^{s}\left(\begin{array}{c}
s+t-2 \\
s-1
\end{array}\right)\left(\sigma_{\mathrm{h}}(1, s+t-1)+\zeta(s+t)\right) .
\end{aligned}
$$

We will now distinguish the two cases $s+t$ odd and $s+t$ even. First, we treat the case where $s+t=2 n+1$. We have $2 n-2$ equations in the $2 n-2$ unknowns $\sigma_{h}(2,2 n-1)$, $\sigma_{\mathrm{h}}(3,2 n-2), \ldots, \sigma_{\mathrm{h}}(2 n-1,2)$. We can reduce the $\sigma_{\mathrm{h}}(k, 2 n+1-k)$ with $k>n$ to $\sigma_{\mathrm{b}}(2 n+1-k, k)$ by the reflection formulas. This leaves us with the $n-1$ unknowns $\sigma_{\mathrm{h}}(2,2 n-1), \ldots, \sigma_{\mathrm{h}}(n, n+1)$. The matrix which corresponds to these equations has the entries

$$
\left(A_{j}^{(k, 2 n+1-k)}+B_{j}^{(k, 2 n+1-k)}-B_{2 n+1-k}^{(k, 2 n+1-k)}\right)_{j, k=2, \ldots, n}
$$

However, it will simplify matters considerably if we augment this matrix by allowing $j, k$ to run from 1 to $n$, and then multiply alternate rows by -1 .

Define, therefore, the $n \times n$ matrices $A, B, C, M$ by

$$
A_{k j}:=(-1)^{k+1}\left(\begin{array}{c}
2 n-j \\
2 n-k
\end{array}\right), B_{k j}:=(-1)^{k+1}\left(\begin{array}{c}
2 n-j \\
k-1
\end{array}\right), C_{k j}:=(-1)^{k+1}\left(\begin{array}{c}
j-1 \\
k-1
\end{array}\right)
$$

$(k, j=1, \ldots, n)$ and $M:=A+B-C$. Define the $n$ vector $r$ by $r_{1}:=0$ and

$$
r_{k}:=(-1)^{k+1}\left[\zeta(k) \zeta(2 n+1-k)+\sum_{i=k}^{n}\left(\begin{array}{c}
i-1 \\
k-1
\end{array}\right)(\zeta(2 n+1)-\zeta(2 n+1-i) \zeta(i))\right]
$$

for $k=2, \ldots, n$. We then have to solve the system $M x=r$ where the vector $x$ has $x_{1}:=0$, and $x_{k}:=\sigma_{\mathrm{h}}(k, 2 n+1-k)$ for $k>1$.

We need the following lemma which is known and can be proved by induction on $m$. 
Lemma 2. (i) For $0 \leqq \mu \leqq m$,

$$
\sum_{i=0}^{\mu}\left(\begin{array}{c}
m-\mu+i \\
i
\end{array}\right)=\left(\begin{array}{c}
m+1 \\
\mu
\end{array}\right)
$$

(ii) For $0 \leqq v, \mu \leqq m-1$,

$$
\sum_{i=1}^{m}(-1)^{i+1}\left(\begin{array}{c}
m-i \\
v
\end{array}\right)\left(\begin{array}{c}
m-\mu-1 \\
i-1
\end{array}\right)=\left(\begin{array}{c}
\mu \\
m-v-1
\end{array}\right)
$$

Setting $\mu=0$ in (ii) and changing the order of summation yields

$$
\sum_{i=1}^{m}(-1)^{i+1}\left(\begin{array}{c}
i-1 \\
v-1
\end{array}\right)\left(\begin{array}{c}
m-1 \\
i-1
\end{array}\right)=(-1)^{m+1} \delta_{m v}
$$

Lemma 2(ii) will now be used to prove the following matrix identities.

$$
\begin{gathered}
A^{2}=C^{2}=I, \\
A=B C, \quad B=A C, \quad C=A B, \\
B^{2}=C A, \quad C B=B A .
\end{gathered}
$$

(It follows from these identities that $B^{3}=B C A=A A=I$, and that the matrix group generated by $A, B$ and $C$ is the permutation group on 3 symbols, but we will not use this.)

\section{Proof of (7a).}

$$
\begin{aligned}
\left(A^{2}\right)_{k j} & =(-1)^{k+1} \sum_{i=1}^{n}(-1)^{i+1}\left(\begin{array}{c}
2 n-i \\
2 n-k
\end{array}\right)\left(\begin{array}{c}
2 n-j \\
2 n-i
\end{array}\right) \\
& =(-1)^{k+1} \sum_{i=n+1}^{2 n}(-1)^{i}\left(\begin{array}{c}
i-1 \\
2 n-k
\end{array}\right)\left(\begin{array}{c}
2 n-j \\
i-1
\end{array}\right)=(-1)^{k+1}(-1)^{j+1} \delta_{k j}
\end{aligned}
$$

Proof of (7b).

$$
\left(C^{2}\right)_{k j}=(-1)^{k+1} \sum_{i=1}^{n}(-1)^{i+1}\left(\begin{array}{c}
i-1 \\
k-1
\end{array}\right)\left(\begin{array}{c}
j-1 \\
i-1
\end{array}\right)=(-1)^{k+1}(-1)^{j+1} \delta_{k j}
$$

\section{Proof of (8a).}




$$
(B C)_{k j}=(-1)^{k+1} \sum_{i=1}^{n}(-1)^{i+1}\left(\begin{array}{c}
2 n-i \\
k-1
\end{array}\right)\left(\begin{array}{c}
j-1 \\
i-1
\end{array}\right)=(-1)^{k+1}\left(\begin{array}{c}
2 n-j \\
2 n-k
\end{array}\right)
$$

Equation (8b) follows from (8a) and (7): $A C=B C^{2}=B$, and similarly $A B=A A C=C$ which is $(8 \mathrm{c})$.

\section{Proof of (9a):}

$$
\begin{aligned}
\left(B^{2}\right)_{k j}-(C A)_{k j}= & (-1)^{k+1} \sum_{i=1}^{n}(-1)^{i+1}\left(\begin{array}{c}
2 n-i \\
k-1
\end{array}\right)\left(\begin{array}{c}
2 n-j \\
i-1
\end{array}\right) \\
& -(-1)^{k+1} \sum_{i=1}^{n}(-1)^{i+1}\left(\begin{array}{c}
i-1 \\
k-1
\end{array}\right)\left(\begin{array}{c}
2 n-j \\
2 n-i
\end{array}\right) \\
= & (-1)^{k+1} \sum_{i=1}^{n}(-1)^{i+1}\left(\begin{array}{c}
2 n-i \\
k-1
\end{array}\right)\left(\begin{array}{c}
2 n-j \\
i-1
\end{array}\right) \\
& +(-1)^{k+1} \sum_{i=n+1}^{2 n}(-1)^{i+1}\left(\begin{array}{c}
2 n-i \\
k-1
\end{array}\right)\left(\begin{array}{c}
2 n-j \\
i-1
\end{array}\right) \\
= & (-1)^{k+1}\left(\begin{array}{c}
j-1 \\
2 n-k
\end{array}\right)=0 .
\end{aligned}
$$

Equation (9b) follows from the other identities: $C B=A B^{2}=A C A=B A$.

These matrix identities now allow us to show that $M$ is invertible; in fact, we have

$$
\begin{gathered}
M^{2}=A A+A B-A C+B A+B B-B C-C A-C B+C C \\
=I+C-B-A+I=2 I-M
\end{gathered}
$$

so that

$$
M^{-1}=\frac{1}{2}(M+I) \text {. }
$$

Thus, to prove Euler's formula, it remains to determine $M^{-1} r$. For this purpose, define $p_{1}:=p_{2 n}:=0, \quad p_{k}:=\zeta(k) \zeta(2 n+1-k) \quad$ for $\quad k=2, \ldots, 2 n-1, \quad p:=\left(p_{k}\right)_{k=1, \ldots, n}, \quad \bar{p}:=$ $\left((-1)^{k+1} p_{k}\right)_{k=1, \ldots, n}$ and $e:=(1, \ldots, 1)$. Then $r_{k}=\bar{p}_{k}-(C p)_{k}+\zeta(2 n+1)(C e)_{k}$. Now let $k \geqq 2$. Then it follows that 


$$
\begin{aligned}
\left(M^{-1} r\right)_{k}= & \left(\frac{1}{2}(M+I) r\right)_{k} \\
= & \frac{1}{2}\left[((A+B-C+I) \bar{p})_{k}-((A+B-C+I) C p)_{k}\right. \\
& \left.+\zeta(2 n+1)((A+B-C+I) C e)_{k}\right] \\
= & \frac{1}{2}\left[((A+B-C+I) \bar{p})_{k}-((A+B+C-I) p)_{k}\right. \\
& \left.+\zeta(2 n+1)((A+B+C-I) e)_{k}\right] .
\end{aligned}
$$

Now, we have

$$
\begin{aligned}
& (A p)_{k}=(-1)^{k+1} \sum_{i=1}^{n}\left(\begin{array}{c}
2 n-i \\
2 n-k
\end{array}\right) p_{i}=(-1)^{k+1} \sum_{i=1}^{2 n}\left(\begin{array}{c}
i-1 \\
2 n-k
\end{array}\right) p_{i} \\
& (B p)_{k}=(-1)^{k+1} \sum_{i=1}^{n}\left(\begin{array}{c}
2 n-i \\
k-1
\end{array}\right) p_{i}=(-1)^{k+1} \sum_{i=n+1}^{2 n}\left(\begin{array}{c}
i-1 \\
k-1
\end{array}\right) p_{i} \\
& (A \bar{p})_{k}=(-1)^{k+1} \sum_{i=1}^{n}(-1)^{i+1}\left(\begin{array}{c}
2 n-i \\
2 n-k
\end{array}\right) p_{i}=-(-1)^{k+1} \sum_{i=1}^{2 n}(-1)^{i+1}\left(\begin{array}{c}
i-1 \\
2 n-k
\end{array}\right) p_{i} \\
& (B \bar{p})_{k}=(-1)^{k+1} \sum_{i=1}^{n}(-1)^{i+1}\left(\begin{array}{c}
2 n-i \\
k-1
\end{array}\right) p_{i}=-(-1)^{k+1} \sum_{i=n+1}^{2 n}(-1)^{i+1}\left(\begin{array}{c}
i-1 \\
k-1
\end{array}\right) p_{i}
\end{aligned}
$$

and therefore

$$
\begin{gathered}
(A \tilde{p}-A p)_{k}=-(-1)^{k+1} \sum_{i=1}^{2 n}\left((-1)^{i+1}+1\right)\left(\begin{array}{c}
i-1 \\
2 n-k
\end{array}\right) p_{i} \\
=2(-1)^{k} \sum_{j=2}^{n}\left(\begin{array}{c}
2 j-2 \\
2 n-k
\end{array}\right) \zeta(2 j-1) \zeta(2 n-2 j+2) \\
((B-C) \bar{p}-(B+C) p)_{k}=-(-1)^{k+1} \sum_{i=1}^{2 n}\left((-1)^{i+1}+1\right)\left(\begin{array}{c}
i-1 \\
k-1
\end{array}\right) p_{i} \\
=2(-1)^{k} \sum_{j=2}^{n}\left(\begin{array}{c}
2 j-2 \\
k-1
\end{array}\right) \zeta(2 j-1) \zeta(2 n-2 j+2) .
\end{gathered}
$$

We also have, by Lemma 2(i), 


$$
\begin{aligned}
(A e)_{k} & =(-1)^{k+1} \sum_{i=1}^{n}\left(\begin{array}{c}
2 n-i \\
2 n-k
\end{array}\right)=(-1)^{k+1} \sum_{i=0}^{k-1}\left(\begin{array}{c}
2 n-k+i \\
i
\end{array}\right) \\
& =(-1)^{k+1}\left(\begin{array}{c}
2 n \\
k-1
\end{array}\right), \\
((B+C) e)_{k} & =(-1)^{k+1} \sum_{i=1}^{n}\left(\begin{array}{c}
2 n-i \\
k-1
\end{array}\right)+(-1)^{k+1} \sum_{i=1}^{n}\left(\begin{array}{c}
i-1 \\
k-1
\end{array}\right) \\
& =(-1)^{k+1} \sum_{i=n-k+1}^{2 n-k}\left(\begin{array}{c}
i+k-1 \\
i
\end{array}\right)+(-1)^{k+1} \sum_{i=0}^{n-k}\left(\begin{array}{c}
i+k-1 \\
i
\end{array}\right) \\
& =(-1)^{k+1}\left(\begin{array}{c}
2 n \\
k
\end{array}\right) .
\end{aligned}
$$

Now, combining equation (10) with the evaluations (11)-(14), we get

$$
\begin{aligned}
2 \sigma_{\mathrm{h}}(k, 2 \mathrm{n}+1-k) & =2\left(M^{-1} r\right)_{k} \\
& =(-1)^{k} 2 \sum_{j=2}^{n}\left[\left(\begin{array}{c}
2 j-2 \\
k-1
\end{array}\right)+\left(\begin{array}{c}
2 j-2 \\
2 n-k
\end{array}\right)\right] \zeta(2 j-1) \zeta(2 n-2 j+2) \\
& +\left((-1)^{k+1}+1\right) \zeta(k) \zeta(2 n+1-k)+\left((-1)^{k+1}\left(\begin{array}{c}
2 n+1 \\
k
\end{array}\right)-1\right) \zeta(2 n+1)
\end{aligned}
$$

from which Euler's formula follows (with the use of the reflection formulas for $\left.\sigma_{\mathrm{h}}(n+1, n), \ldots, \sigma_{\mathrm{h}}(2 n-1,2)\right)$.

In the even case, $s+t=2 n$, the equations will in general not be uniquely solvable, except for $n=2$ and $n=3$. In these cases, our reflection and decomposition formulas, together with (3), immediately yield the asserted values for $\sigma_{h}(2,2)$ and $\sigma_{h}(2,4)$. For $n>3$, however, the corresponding $(n-1) \times(n-1)$ matrix $\tilde{M}=\tilde{A}+\widetilde{B}-\tilde{C}$, with

$$
\tilde{A}_{k j}:=(-1)^{k+1}\left(\begin{array}{l}
2 n-j-1 \\
2 n-k-1
\end{array}\right), \quad \tilde{B}_{k j}:=(-1)^{k+1}\left(\begin{array}{c}
2 n-j-1 \\
k-1
\end{array}\right)
$$




$$
\tilde{C}_{k j}:=(-1)^{k+1}\left(\begin{array}{l}
j-1 \\
k-1
\end{array}\right), \text { for } j, k=1, \ldots, n-1 \text {, }
$$

has minimal polynomial $\tilde{M}^{3}+2 \tilde{M}^{2}-3 \tilde{M}=0$. [Note that $\sigma_{\mathrm{h}}(n, n)$ evaluates directly.] This may be proved in much the same way as in the odd case. It follows from analysis of the trace of $\tilde{M}$ and of $\tilde{M}^{2}$ that the number of null eigenvalues is $\lfloor(n-1) / 3\rfloor$ and, since the minimal polynomial has no repeated roots, that the dimension of the null space is $\lfloor(n-1) / 3\rfloor$.

\section{Alternating sums}

The same technique that allows us to evaluate the constants $\sigma_{\mathrm{h}}(s, t)$ can be used for the alternating analogues $\alpha_{\mathrm{h}}(s, t), \sigma_{\mathrm{a}}(s, t), \alpha_{\mathrm{a}}(s, t)$. Define

$$
\eta(z):=\sum_{n=1}^{\infty} \frac{(-1)^{n-1}}{n^{z}}=\left(1-2^{1-z}\right) \zeta(z) .
$$

Then we have the following equations:

$$
\begin{gathered}
\alpha_{\mathrm{h}}(s, t)-\sigma_{\mathrm{a}}(t, s)=\eta(s+t)-\zeta(s) \eta(t), \quad s \geqq 2, t \geqq 1, \\
\alpha_{\mathrm{a}}(s, t)+\alpha_{\mathrm{a}}(t, s)=\zeta(s+t)-\eta(s) \eta(t), \quad s, t \geqq 1, \\
\eta(s) \eta(t)=\sum_{j=1}^{s} A_{j}^{(s, t)} \alpha_{\mathrm{h}}(j, s+t-j)+\sum_{j=1}^{t} B_{j}^{(s, t)} \alpha_{\mathrm{h}}(j, s+t-j), \quad s, t \geqq 1, \\
\eta(s) \zeta(t)=\sum_{j=1}^{s} A_{j}^{(s, t)} \sigma_{\mathrm{a}}(j, s+t-j)+\sum_{j=1}^{t} B_{j}^{(s, t)} \alpha_{\mathrm{a}}(j, s+t-j), \quad s \geqq 1, t \geqq 2 .
\end{gathered}
$$

The use of $\eta$ instead of $\zeta$ alone greatly simplifies the form of these equations. The two reflection formulas can be found in Nielsen's book [8] on page 47, while Nielsen uses slightly different decomposition formulas. He uses these to prove, e.g.,

$$
2 \sigma_{\mathrm{a}}(1, m)=2 \eta(1) \zeta(m)-m \zeta(m+1)+\sum_{k=1}^{m} \eta(k) \eta(m+1-k)
$$

(follows from formula (6), p. 50 in [8], by use of the first reflection formula), and

$$
\alpha_{\mathrm{b}}(1, m)-\alpha_{\mathrm{a}}(1, m)=\eta(1) \eta(m)-m \eta(m+1)+\sum_{k=0}^{m-2} \eta(k+1) \zeta(m-k)
$$

(follows from formula (9), p. 50 in [8], by use of the second reflection formula). 
We will need

$$
\alpha_{\mathrm{a}}(1, m)=m \eta(m+1)-\sum_{k=1}^{m-1} \eta(k) \zeta(m+1-j)-\sum_{j=1}^{m} \alpha_{\mathrm{h}}(m+1-j, j),
$$

which follows from equations (10), p. 48 and (9), p. 50 in [8].

Again, if $s+t$ is even, only a few of the series can be evaluated by solving these equations. For example

$$
\begin{aligned}
& \alpha_{h}(1,1)=\frac{\ln ^{2}(2)}{2} \\
& \alpha_{a}(1,1)=\frac{\zeta(2)-\ln ^{2}(2)}{2} .
\end{aligned}
$$

Moreover, the following evaluations can be proved using formulas in [7] (a mistranscribed version of these is given in $[10$, p. 3]):

$$
\begin{aligned}
& \alpha_{\mathrm{h}}(1,3)=2 \mathrm{Li}_{4}\left(\frac{1}{2}\right)+\frac{\ln ^{4}(2)}{12}-\frac{15 \zeta(4)}{8}+\frac{7 \zeta(3) \ln (2)}{4}-\frac{\zeta(2) \ln ^{2}(2)}{2} \\
& \alpha_{\mathrm{a}}(1,3)=2 \mathrm{Li}_{4}\left(\frac{1}{2}\right)+\frac{\ln ^{4}(2)}{12}+\frac{3 \zeta(4)}{4}-\frac{\zeta^{2}(2)}{2}-\frac{\zeta(2) \ln ^{2}(2)}{2}
\end{aligned}
$$

These formulas are equivalent in light of equation (15). We believe that for larger odd $m$ the sums $\alpha_{h}(1, m)$ do not evaluate in terms of zeta functions or special values of polylogarithms (see [1]).

We now turn to the case where $s+t$ is odd. If $s+t=2 n+1$, then we have the unknowns

$$
\begin{gathered}
\alpha_{\mathrm{b}}(1,2 n), \ldots, \alpha_{\mathrm{b}}(n, n+1), \alpha_{\mathrm{b}}(n+1, n), \ldots, \alpha_{\mathrm{b}}(2 n-1,2), \alpha_{\mathrm{b}}(2 n, 1), \\
\sigma_{\mathrm{a}}(1,2 n), \ldots, \sigma_{\mathrm{a}}(n, n+1), \sigma_{\mathrm{a}}(n+1, n), \ldots, \sigma_{\mathrm{a}}(2 n-1,2), \\
\alpha_{\mathrm{a}}(1,2 n), \ldots, \alpha_{\mathrm{a}}(n, n+1), \alpha_{\mathrm{a}}(n+1, n), \ldots, \alpha_{\mathrm{a}}(2 n-1,2), \alpha_{\mathrm{a}}(2 n, 1),
\end{gathered}
$$

and the equations

$$
\begin{array}{ll}
\alpha_{\mathrm{b}}(2 n+1-k, k)=\sigma_{\mathrm{a}}(k, 2 n+1-k)+\eta(2 n+1)-\eta(k) \zeta(2 n+1-k) & \text { for } k=1, \ldots, n, \\
\sigma_{\mathrm{a}}(2 n+1-k, k)=\alpha_{\mathrm{b}}(k, 2 n+1-k)-\eta(2 n+1)+\zeta(k) \eta(2 n+1-k) & \text { for } k=2, \ldots, n, \\
\alpha_{\mathrm{a}}(2 n+1-k, k)=-\alpha_{\mathrm{a}}(k, 2 n+1-k)+\zeta(2 n+1)-\eta(k) \eta(2 n+1-k) & \text { for } k=1, \ldots, n,
\end{array}
$$




$$
\zeta(k) \eta(2 n+1-k)=\sum_{j=1}^{k} A_{j}^{(k, 2 n+1-k)} \alpha_{\mathrm{a}}(j, 2 n+1-j)+\sum_{j=1}^{2 n+1-k} B_{j}^{(k, 2 n+1-k)} \sigma_{\mathrm{a}}(j, 2 n+1-j)
$$

for $k=2, \ldots, n$,

$$
\eta(k) \eta(2 n+1-k)=\sum_{j=1}^{k} A_{j}^{(k, 2 n+1-k)} \alpha_{\mathrm{h}}(j, 2 n+1-j)+\sum_{j=1}^{2 n+1-k} B_{j}^{(k, 2 n+1-k)} \alpha_{\mathrm{h}}(j, 2 n+1-j)
$$

for $k=1, \ldots, n$,

$$
\eta(k) \zeta(2 n+1-k)=\sum_{j=1}^{k} A_{j}^{(k, 2 n+1-k)} \sigma_{\mathrm{a}}(j, 2 n+1-j)+\sum_{j=1}^{2 n+1-k} B_{j}^{(k, 2 n+1-k)} \alpha_{\mathrm{a}}(j, 2 n+1-j)
$$

for $k=1, \ldots, n$,

Obviously, we have one equation less than the number of unknowns, so we need an additional equation. As it turns out, the equation which will naturally occur here is

$$
\sum_{j=1}^{n} \alpha_{\mathrm{h}}(j, 2 n+1-j)+\sum_{j=1}^{n} \sigma_{\mathrm{a}}(j, 2 n+1-j)+\alpha_{\mathrm{a}}(1,2 n)=n \eta(2 n+1)-\sum_{j=2}^{n} \zeta(j) \eta(2 n+1-j)
$$

which by the refection formulas is equvalent to equation (16).

By substituting the reflection formulas into the decomposition equations we can reduce the number of unknowns to the $3 n$ unknowns

$$
\alpha_{\mathrm{b}}(k, 2 n+1-k), \sigma_{\mathrm{a}}(k, 2 n+1-k), \alpha_{\mathrm{a}}(k, 2 n+1-k) \text { for } \quad k=1, \ldots, n
$$

The matrix which corresponds to the resulting equations (after multiplying alternative equations by -1 ) is the matrix $N \in \mathbb{R}^{3 n \times 3 n}$ which is given by

$$
N:=\left(\begin{array}{ccc}
C & B & A \\
A+B & C & 0 \\
0 & A & B-C
\end{array}\right)
$$

The vector on the right-hand side is the vector $r \in \mathbb{R}^{3 n}$ with the entries

$$
\begin{gathered}
r_{1}:=n \eta(2 n+1)-\sum_{j=2}^{n} \zeta(j) \eta(2 n+1-j), \\
r_{k}:=(-1)^{k+1}\left(\zeta(k) \eta(2 n+1-k)+\sum_{j=k}^{n}\left(\begin{array}{c}
j-1 \\
k-1
\end{array}\right)(\eta(2 n+1)-\zeta(j) \eta(2 n+1-j))\right)
\end{gathered}
$$

for $k=2, \ldots, n$; 


$$
\begin{aligned}
& r_{k+n}:=(-1)^{k+1}\left(\eta(k) \eta(2 n+1-k)-\sum_{j=k}^{n}\left(\begin{array}{l}
j-1 \\
k-1
\end{array}\right)(\eta(2 n+1)-\eta(j) \zeta(2 n+1-j))\right), \\
& r_{k+2 n}:=(-1)^{k+1}\left(\eta(k) \zeta(2 n+1-k)-\sum_{j=k}^{n}\left(\begin{array}{l}
j-1 \\
k-1
\end{array}\right)(\zeta(2 n+1)-\eta(j) \eta(2 n+1-j))\right),
\end{aligned}
$$

for $k=1, \ldots, n$.

The very first equation is exactly the additional equation we introduced earlier.

This system has a unique solution, because the matrix $N$ is invertible; indeed the inverse is

$$
N^{-1}=\frac{1}{2}\left(\begin{array}{ccc}
C-I & A & -B \\
0 & C-B & A+I \\
A+B & -I & -C
\end{array}\right)
$$

as can be shown using the matrix identities (7)-(9) for the matrices $A, B, C$. Now, one could give explicit formulas for $\alpha_{\mathrm{h}}(s, t), \sigma_{\mathrm{a}}(s, t), \alpha_{\mathrm{a}}(s, t)$ by applying $N^{-1}$ to $r$. However, things do not simplify quite as nicely as in the non-alternating case. We therefore choose to leave matters in this somewhat more implicit state. Let us just remark that the following formulas can be proved in that way:

$$
\begin{gathered}
2 \alpha_{\mathrm{b}}(1,2 n)=\zeta(2 n+1)-(2 n-1) \eta(2 n+1)+2 \sum_{k=1}^{n-1} \eta(2 k) \zeta(2 n+1-2 k), \\
2 \alpha_{\mathrm{a}}(1,2 n)=\zeta(2 n+1)+(2 n+1) \eta(2 n+1)-2 \eta(1) \eta(2 n)-2 \sum_{k=1}^{n} \zeta(2 k) \eta(2 n+1-2 k) .
\end{gathered}
$$

These were proved by $R$. Sitaramachandra Rao in [10]; he gives them in a more complicated fashion than we do here, though.

Finally, we note that D. Zagier (private communication) has informed us that he is studying the modular-function properties of $\sigma_{\mathrm{h}}(s, t)$. His work provides, inter alia, an alternative and elegant abstract method of showing that the $\sigma_{\mathrm{h}}(s, t)$ must evaluate in terms of zeta functions when $s+t$ is odd. 
Note added in proof. It came to our attention recently that in "Triple sums and the Riemann zeta function," J. Number Theory 48 (1994), 113-132, C. Markett proved that

$$
\begin{gathered}
\sum_{0<n_{1}<n_{2}<n_{3}} \frac{1}{n_{1} \cdot n_{2} \cdot n_{3}^{m}}=\frac{m(m+1)}{6} \zeta(m+2)-\frac{m-1}{2} \zeta(2) \zeta(m) \\
-\frac{m}{4} \sum_{k=0}^{m-4} \zeta(m-k-1) \zeta(k+3)+\frac{1}{6} \sum_{k=0}^{m-4} \zeta(m-k-2) \sum_{j=0}^{k} \zeta(k-j+2) \zeta(j+2) .
\end{gathered}
$$

In the light of the formula

$$
s_{\mathrm{h}}(2, m)=2 \sum_{0<n_{1}<n_{2}<n_{3}} \frac{1}{n_{1} \cdot n_{2} \cdot n_{3}^{m}}+\sigma_{\mathrm{h}}(2, m)
$$

this is equivalent to our formula (2).

\section{REFERENCES}

1. D. H. Balley, J. Borwein and R. Girgensohn, Experimental evaluation of Euler sums, Experiment. Math. 3 (1994), 17-30.

2. B. C. Berndt, Ramanujan's Notebooks, Part I (Springer-Verlag, New York, 1985).

3. D. Borwein, J. M. Borwein, On an intriguing integral and some series related to $\zeta(4)$, Proc. Amer. Math. Soc., to appear.

4. P. J. DE Doelder, On some series containing $\Psi(x)-\Psi(y)$ and $(\Psi(x)-\Psi(y))^{2}$ for certain values of $x$ and $y, J$. Comput. Appl. Math. 37 (1991), 125-141.

5. L. Euler, Opera Omnia, Ser 1, Vol. XV (Teubner, Berlin 1917), 217-267.

6. M. Hoffman, Multiple harmonic series, Pacific J. Math. 152 (1992), 275-290.

7. L. Lewin, Polylogarithms and Associated Functions (North-Holland, New York, 1981).

8. N. Nielsen, Die Gammafunktion (Chelsea, New York 1965).

9. R. Sitaramachandra Rao, M. V. Subbarao, Transformation formulae for multiple series, Pacific J. Math. 113 (1984), 471-479.

10. R. Sitaramachandra Rao, A formula of S. Ramanujan, J. Number Theory 25 (1987), 1-19.

11. K. R. Stromberg, An Introduction to Classical Real Analysis (Wadsworth, 1981).

David Borwein

Department of Mathematics

UNIVERSITY OF WESTERN ONTARIO

London. Ontario N6A 5B7

CANADA

Roland Girgensohn

Department of Pure Mathematics

UNIVERSITY OF WATERLOO

Waterloo. Ontario N2L 3G1

CAnAda
Jonathan M. Borwein

Department of Mathematics and Statistics

SiMON FRASER UNIVERSITY

Burnaby. BC V5A IS6

Canada 